\title{
Student Emotion in Mediated Learning: Comparing a Text, Video, and Video Game
}

\author{
Aubrie Adams ${ }^{1}$ and Weimin Toh ${ }^{2}$ \\ ${ }^{1}$ California Polytechnic State University, San Luis Obispo, USA \\ ${ }^{2}$ National Institute of Education, Nanyang Technological University, Jurong West, Singapore \\ aadams45@calpoly.edu \\ a0019282@u.nus.edu
}

\begin{abstract}
Although serious games are generally praised by scholars for their potential to enhance teaching and e-learning practices, more empirical evidence is needed to support these accolades. Existing research in this area tends to show that gamified teaching experiences do contribute to significant effects to improve student cognitive, motivational, and behavioural learning outcomes, but these effects are usually small. In addition, less research examines how different types of mediated learning tools compare to one another in influencing student outcomes associated with learning and motivation. As such, a question can be asked in this area: how do video games compare to other types of mediated tools, such as videos or texts, in influencing student emotion outcomes? This study used an experimental design $(N=153)$ to examine the influence of different types of mass media modalities (text, video, and video game) on college students' emotions in a mediated learning context. Research examining the impact of video games on instruction has begun to grow, but few studies appropriately acknowledge the nuanced differences between media tools in comparison to one another. Using a media-attributes approach as a lens, this study first compared these mediated tools along the attributional dimensions of textuality, channel, interactivity, and control. This study next tested the impact of each media type on thirteen emotion outcomes. Results showed that six emotion outcomes did not indicate differences between groups (fear, guilt, sadness, shyness, serenity, and general negative emotions). However, six of the tested emotion outcomes did indicate differences between groups with students experiencing higher levels of emotional arousal in both the text and video game conditions (in comparison to the video condition) for the emotions of joviality, self-assurance, attentiveness, surprise, hostility, and general positive emotions. Lastly, students also felt less fatigue in the video game condition. Overall, implications for e-learning suggest that when a message's content is held constant, both video games and texts may be better in inducing emotional intensity and reducing fatigue than videos alone, which could enhance motivation to learn when teaching is mediated by technology.
\end{abstract}

Keywords: emotion, game-based learning, media comparison, motivation, text, video

\section{Introduction}

Technological advances provide new ways for instructors to implement computer-mediated learning opportunities; in particular, serious games have been adapted to facilitate educational processes. Although scholars have praised serious games' potential to enhance teaching and learning practices (Koster, 2004; McGonigal, 2011; Squire, 2011; Schrier, 2016), more empirical evidence and scholarly work is needed to support these accolades (Connolly et al., 2012; Hilton and Honey, 2011; Jacobs, 2021; Mayer, 2014). As such, this study examines how video games compare with other mass media educational technologies in their ability to influence student emotion-related outcomes. Pragmatic research is needed to identify the affordances, limitations, and implications of using serious games in university settings to better understand their impacts on outcomes related to e-learning processes.

This experiment examines three types of mass media tools: text, video, and video game. Each platform varies in its degree of textuality, channel, interactivity, and control. Although research examining video games' effects on instruction continues to grow (Prensky, 2005; Sailer and Homner, 2020; Squire, 2003; Yee, 2013), few studies test the nuanced differences among instructional mass media tools to specifically examine student emotions. Therefore, this study seeks to examine these key differences to explore the implications this may have on student learning. In reviewing existing literature, the following topics are outlined in this paper: (1) an explication of serious games; (2) a broad overview of the mixed attributes approach to media research; and (3) a review of emotion outcomes as they relate to motivation and educational processes.

\section{Background}

\subsection{Serious games}

Scholars have contested the conceptualisation of what is considered a "game" (for a review, see Juul, 2005). One definition purports that games are activities containing six features: (1) rules, (2) variable quantifiable 
outcomes, (3) valorisation of outcomes, (4) player efforts, (5) player outcome attachments, and (6) negotiable consequences (Juul, 2005, p. 8). A broader definition claims that games simply exist as rule-defined activities where players seek to reach goals (Galloway, 2006, p. 19). Between both definitions, the current study utilises and extends the broader conceptualisation, claiming that video games are rule-based activities where players seek to accomplish tasks in digitally constructed environments.

Although many video games function as entertainment and recreational activities, serious games work as training and educational tools (Connolly et al., 2012). Numerous types of serious games are available, including advergames, newsgames, educational, persuasive, health, art, social impact (Grace, 2020), and productivity games. Related to serious games, gamification is a process that uses elements of play to modify and enhance pre-existing educational and training practices. Games are constructed to teach a range of topics, including ethics (Brown, 2006), physics instruction (Jackson, 2011), mitigation of cognitive biases (Dunbar et al., 2013), computer coding (Mandaro, 2014), deception detection skills (Miller et al., 2019), and coping with anxiety (Heumos and Kickmeier-Rust, 2020). A meta-analysis that synthesised research findings on game-based learning effects found overall significant, but relatively small, positive effects on cognitive, motivational, and behavioural learning outcomes (Sailer and Homner, 2020).

In related research on serious games, Ferguson, van den Broek, and van Oostendorp (2020) tested how varying interaction modes (active versus passive) and story structures (explicit versus implicit) affected participants' sense of presence, cognitive interest, and engagement in a virtual reality game. Results showed that allowing players freedom to navigate their gameplay positively influenced their cognitive interests and feelings of presence. Additionally, the use of implicit story structures contributed to the players' increased recall of spatial information. Nonetheless, players in this study still needed guidance to enhance student learning of fact-based knowledge.

Essentially, games offer general benefits to the educational environment and a key reason why is because they may enhance student motivation to learn. Indeed, research on digital games suggests that the lure of game play may motivate an assortment of positive behaviours in tasks that users may not ordinarily be inclined to perform. In this area, augmented reality games have been shown to motivate users to increase their physical activity in games like Pokémon Go (Althoff, White and Horvitz, 2016) and civic games can encourage users to get more involved in participating in community-based projects like those designed to improve local parks (Coulter et al., 2012).

However, despite the affordances that games offer the educational environment, challenges do exist. For example, scholars have questioned whether learning from gameplay will suitably transfer to real life contexts (Shaffer, 2012). In addition, Mayer (2014) performed a review of research on serious games and found that in comparison to traditional teaching methods, serious games did not always provide substantial differences in learning outcomes. As such, modern educators have begun to look more closely at issues such as these to better identify the contexts that games can be used to make learning more effective and to share best practices in games for learning "(Ferdig, Baumgartner and Gandolfi, 2021; Schrier, 2019).

Clearly, serious games have the potential to enhance educational processes to some degree. However, more research is needed to identify the precise mechanisms that affect learning in video games (Sailer and Homner, 2020) and to examine how these mechanisms compare between different types of mass media tools, such as texts or videos. Building on prior research on the use of serious games for mediated learning, this paper offers a mixed attributes approach that enables researchers to investigate how different types of educational technologies comparatively affect learning-related outcomes, such as emotional arousal.

\subsection{Mixed attributes approach to media studies}

According to McLuhan's (1964, p. 2) well-known axiom, "the medium is the message," the vehicle or type of tool that is used to deliver a message can have a substantial impact on how that message is perceived and the subsequent information processing of that message. This truism suggests that even when a message's content is held constant, different types of media tools likely contribute to varying outcomes (Detenber and Lang, 2010). From this perspective, researchers can examine a range of educational technologies that contribute differently to teaching and learning effects. For example, distance learning options from the past and present (for a review, see Moore, 2013) have included various media tools, such as mail correspondence (Bittner and Mallory, 1933), television broadcasting (Benschoter and Charles, 1957), and internet discussion boards 
(Howland and Moore, 2002). Given these tools and newer educational technologies, such as serious games, it is reasonable to predict substantial variations in the quality of teaching and learning afforded and limited by each media type. As such, this paper uses a media effects perspective to examine the ways in which learning outcomes may vary depending on the type of media tool used to deliver educational content.

In drawing on a media effects perspective, Eveland (2003) describes a mixed attributes approach that scholars can use to analyse specific outcomes induced by different media types. This approach proposes a framework to conceptualise, explicate, and compare media to better understand the underlying mechanisms that affect the outcomes directly resulting from differences among media types. This framework differs from earlier media effects research in three primary ways. First, the mixed attributes approach does not solely rely on the qualitative categorisations of different media forms (e.g., books as a category compared with videos as a category); instead, it uses more quantitative descriptions to assess the specific attributes of each medium along a spectrum. Eveland (2003, p. 398) outlines six related attributes: (1) interactivity, (2) structure, (3) control, (4) sensory channel, (5) textuality, and (6) content. The key to applying a mixed attributes approach is recognising that different media often vary to some degree in these attributes (e.g., books feature more textuality than videos).

Second, this approach assumes that media tools feature multidimensional attributes. For this reason, researchers should not focus on one attribute per tool but examine attribute groupings (e.g., videos feature lower textuality, more channel visualisations, less interaction, and less control). Third, this approach situates modern findings in a historical context. For example, instead of describing a phenomenon such as internet surfing as new, researchers can draw on earlier research on television channel surfing to explore the implications of this media-related behaviour. Therefore, even new educational technologies can be described under existing conceptualisations to some degree.

Utilising Eveland's (2003) mixed attributes approach, this study compares three educational tools (i.e., text, video, video game) and focuses on four of the six media attributes: (1) textuality, (2) sensory channel, (3) interactivity, and (4) control. Structure and content are not compared because these attributes remain constant across the media experiences tested in this research. Additionally, a complete review of all four media attributes is beyond the scope of this investigation (for a more thorough review, see Adams, 2016). Nonetheless, a summary is provided so unfamiliar readers may understand the nuanced differences in media attributes in the text, video, and video game used in this study.

\subsubsection{Textuality}

Textuality refers to the degree that textual symbols (e.g., words) are used in media. Although textuality is primarily interpreted through the visual sense, it can also be interpreted via tactile channels, such as Braille (Eveland, 2003). Books and newspapers typically feature a higher degree of textuality compared with videos and video games. Although some videos and video games heavily rely on communicating through text, many modern video games typically depend little on text components. Therefore, textbooks are typically rated higher in textuality than videos and video games.

\subsubsection{Sensory channel}

Sensory channel refers to the degree that a person's five senses are engaged by a media experience. Typically, many media tools activate a person's senses of sight and sound (Eveland, 2003). Still, regarding sight, textual media are limited because they only activate low visual sensory levels using one-dimensional pictures compared with videos that fundamentally exist as continuous audio and video streams (Lang, 2006). Similarly, video games afford rich audio-video experiences and dynamic interactive events that enable a higher degree of social presence (Kaplan and Haenlein, 2010; Tamborini and Skalski, 2006). Depending on the media type used, a person's ability to experience different levels of sensory information may affect learning-related outcomes in various ways. From this perspective, videos and video games typically engage a person's sensory channels more than texts.

\subsubsection{Interactivity}

The degree of interactivity refers to the extent that a medium allows for participant feedback and response (Eveland, 2003; Newhagen and Rafaeli, 1996). Because a user cannot typically alter pre-written texts or videos, both media offer less interactivity. Comparatively, video games enable a higher degree of interactivity. In single-player games, users can interact with the game environment and non-player characters to affect the 
game's outcome in variable ways (Pivec, 2007). Similarly, in multiplayer games, users can collaborate with others to solve problems and attain goals (Upchurch and Wildermuth, 2014; Yee, 2009). As such, video games are rated higher in interactivity than texts and videos.

\subsubsection{Control}

Lastly, control refers to the users' ability to exercise free will during the media experience. This attribute overlaps with interactivity yet remains distinct. Whereas interactivity refers to users' ability to participate in and affect media content, control refers to the degree that they can influence the content's pausing and pacing (Eveland, 2003; Eveland and Dunwoody, 2001). In this way, text offers a higher degree of control because users can read at their own pace, with the options to go backward, skip ahead, and re-read. To some degree, videos also facilitate control when they allow users to pause, rewind, play, and fast-forward. However, in a video shown in a classroom environment, users have little ability to affect the presentation's order or timing. Similarly, video games typically afford players the ability to influence the pacing, interactions, and movements of their gameplay, yet they may be constrained by rules in the digital environment (Upchurch and Wildermuth, 2014). Therefore, in this study, the text condition is rated higher in control compared with videos and video games.

In summary, this study identifies the media attributes of textuality, sensory channel, interactivity, and control to describe how they vary amongst a text, video, and video game. As mentioned, the mixed attributes approach recommends that scholars should examine attribute groupings for each medium. Table 1 (see Method) summarises the different media tools used in this study to display the precise mechanisms and how they vary so scholars can understand what media features contribute to different outcomes. With this perspective in mind, we next describe the outcome variable of interest in this study: emotion.

\subsection{Emotion}

In a meta-analysis that reports empirical outcomes associated with serious games, Connolly et al. (2012, p. 662 ) found that the three most commonly examined outcomes were (1) knowledge acquisition, (2) affective outcomes, and (3) motivational outcomes. Although knowledge acquisition itself is central to learning, affective and motivational components are necessary as well. Sufficient motivation is needed to engage learners in educational processes and emotional aspects often strengthen their motivation to learn (Bigge, 1982). This investigation adds to the body of literature on serious games by extending the research on emotion as a motivational learning outcome and applying a mixed attributes approach.

Emotions are short-lived, internal mental states that vary in intensity; represent evaluative reactions to events, agents, or objects; and direct attention towards external stimuli (Nabi and Green, 2015, p. 142). Two primary perspectives describe different types of emotions: (1) the discrete view that focuses on emotions as unique sets of cognitive appraisals (Frijda, 1986; Lazarus, 1991) and (2) the dimensional view that focuses on emotions as motivational states that vary by levels of valence, arousal, and dominance (Lang et al., 1993). The dimensional perspective claims that experiences of emotion map on to appetitive (wanting to move towards) and aversive (wanting to move away from) motivational systems (Detenber and Lang, 2010). The present study focuses on the dimensional view given its connection to motivational dynamics as students who are more emotionally connected to learning material are thus more likely to be motivated to seek out and actively engage in teaching and learning processes.

As mentioned, the dimensional view describes three aspects of emotions: (1) valence, (2) arousal, and (3) dominance. Valence refers to the degree that an affect response ranges from pleasant to unpleasant (Detenber and Lang, 2010, p. 278), which maps on to the motivation attention-related aspects of approach (positive emotions) and avoidance (negative emotions). Arousal refers to a response that varies along a continuum ranging from thrilled to composed, which maps on to the degree of intensity of the approach and withdrawal motivations. Dominance refers to the perception of stimulus control. Educational literature shows that providing learning opportunities with more choices (high dominance) leads to increased cognitive engagement and positive affect (Kohn, 1993); although increased choice can also have a negative effect on cognitive task performance (Flowerday and Schraw, 2000). Still, less research has found support for the relevance of the dominance component within emotions (Detenber and Lang, 2010). As such, this study focuses on the dimensions of valence (negative to positive) and arousal (less intense to more intense) when examining student emotion outcomes. 
Overall, studies examining emotion in the context of media messages find that the modification of noncontent related features (e.g., making changes to a media message's size, colour, motion, etc.) can influence levels of emotion (Detenber and Reeves, 1996; Reeves et al., 1999). In addition, positive emotions may function as motivating forces that can encourage learners to better engage in educational processes. However, it is unclear which media tool may better contribute to emotion in e-learning contexts. Because texts, videos, and video games vary in their attributional components, this study utilises a mixed attributes approach to examine how each type of media tool differs. It then investigates the differences' subsequent impacts on outcomes related to emotion arousal and valence. Given the existing research on media attributes, a research question (RQ) is posed: Which media tool will contribute to higher levels of positive emotion in an e-learning context?

\section{Method}

This study utilises an experimental design to manipulate each type of media experience (text, video, or video game) to examine the subsequent impact on student emotion. Although the content remains relatively consistent across all three conditions, each type of media experience varies to the degree that textuality, channel, interactivity, and control are present.

\subsection{Participants}

In total, 153 college students from a large western university in the United States of America were recruited via convenience and volunteer sampling techniques and received a nominal amount of course credit for participation. They represented the following demographics: $69.3 \%$ females and $30.7 \%$ males with a mean age of 19.73 years old $(S D=1.59)$. Participants reported their races as $43.8 \%$ White, $20.3 \%$ Hispanic, $15.7 \%$ East Asian, 7.2\% multiracial, 2.6\% Black, 2.6\% Middle Eastern, 2.6\% South Asian, and 5.2\% did not report a racial category.

\subsection{Procedure}

The participants were directed to an online questionnaire hosted by SurveyGizmo. The questionnaire started with a consent form notifying respondents of their voluntary and confidential participation. Second, they were randomly assigned to one of three stimulus media conditions (text, video, or video game). The participants read the instructions for their specified condition and then either read a narrative, watched a video, or played a video game. The content was controlled across all conditions. Next, the participants completed outcome measures of emotion and additional measures unrelated to the RQ. Lastly, they answered demographic questions.

\subsubsection{Stimulus: video game}

In the video game condition $(n=58)$, the participants played a short digital game: The End of Us (www.theend-of-us.com/), created by Molinari and Howe for the 2011 Global Game Jam (see figure 1). The game lasts approximately four minutes with rudimentary interaction (as players can only control the direction of forward movement). The game builds a simple narrative using a metaphor to explore themes of companionship, life, and death. The player controls a purple comet that flies through outer space. Conflict occurs when an orange comet arrives, with no explanation of its motives. The game has a mostly pre-determined outcome, except for the player's choice that determines which comet survives at the end (either the purple or orange comet). Ultimately, the game's relatively minimalistic style of gameplay is well-suited to compare outcomes across media modalities. 

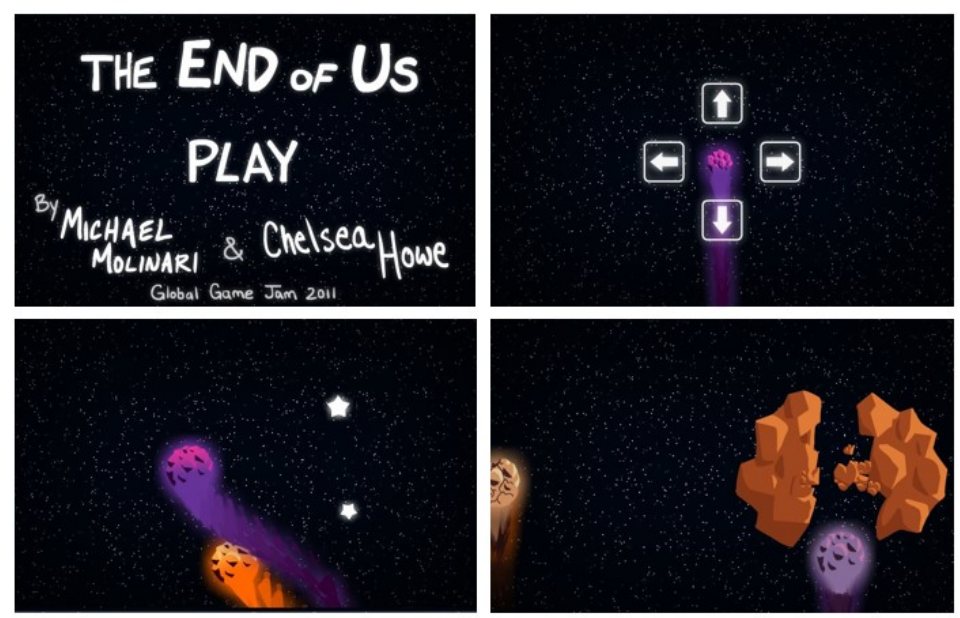

Figure 1: Screenshots for the video game, The End of Us

\subsubsection{Stimulus: video}

In the video condition $(n=48)$, the participants viewed a recording of the game's play-through. Thus, the video game and the video condition stimuli were similar (except that the participants in the video condition could not interact in the game environment or control the final outcome). This method allowed for a realistic comparison between the two modalities with the content between them held reasonably constant.

\subsubsection{Stimulus: text}

Using the recorded video, a description of the observable action in the video game was translated into a written description for the text condition $(n=47)$. The text described the action that occurred in the game and the video. As such, the video game, the video, and the text stimuli were similar, except that the participants in the text condition could not interact in the game environment and lacked a pictorial experience in the visual sensory channel. However, they could read the text description. Additionally, the same music played in each condition to control this variable across the three media types, again facilitating a reasonable comparison. Given the specific video game, video, and text generated for this study, users can apply a media-attributes perspective to examine how each modality differed. Table 1 summarises these differences.

Table 1: Summary of media attributes for text, video, and video game

\begin{tabular}{lcccc}
\hline Media & Textuality & Sensory channel & Interactivity & Control \\
\hline Text & High & Low & Low & High \\
Video & Low & High & Low & Low \\
Video game & Low & High & High & Medium \\
\hline
\end{tabular}

\subsection{Measures of emotion}

This study utilised a modified version of Watson and Clark's (1999) Positive and Negative Affect Schedule (PANAS). The PANAS is a self-report measure that assesses four broad categories of emotion: (1) general dimensions (negative and positive affect); (2) basic negative emotions (fear, hostility, guilt, and sadness); (3) basic positive emotions (joviality, self-assurance, and attentiveness); and (4) other affective states (shyness, fatigue, serenity, and surprise), totalling 13 emotion types.

This study used these 13 emotion types under the four original categories. Individual emotion items were averaged to form composite measures for each type of emotional state. Although the PANAS typically comprises 60 emotion items, this study added 9 additional items. Semantic deferential scales (1-7, from "not at all" to "extremely" experienced) were used to measure all emotions. Each item was randomised in a different order for all participants. Table 2 summarises the emotion categories and the individual emotion items used for each measure. Each emotion measure had acceptable reliability (see Table 3). 
Table 2: PANAS outcome measures; additional variables added for this study marked with an asterisk $\left({ }^{*}\right)$

\begin{tabular}{|c|c|c|}
\hline Category & Emotion & $\begin{array}{ll}\text { Individual emotion items } \\
\end{array}$ \\
\hline General & $\begin{array}{l}\text { 1. Negative } \\
\text { 2. Positive }\end{array}$ & $\begin{array}{l}\text { afraid, scared, nervous, jittery, irritable, hostile, guilty, ashamed, upset, distressed } \\
\text { active, alert, attentive, determined, enthusiastic, excited, inspired, interested, } \\
\text { proud, strong, pleased* }\end{array}$ \\
\hline Negative & $\begin{array}{l}\text { 3. Fear } \\
\text { 4. Hostility } \\
\text { 5. Guilt } \\
\text { 6. Sadness }\end{array}$ & $\begin{array}{l}\text { afraid, scared, frightened, nervous, jittery, shaky } \\
\text { angry, hostile, irritable, scornful, disgusted, loathing, annoyed*, frustrated* } \\
\text { guilty, ashamed, blameworthy, angry at self, disgusted w/self, dissatisfied w/self, } \\
\text { remorseful* } \\
\text { sad, blue, downhearted, alone, lonely }\end{array}$ \\
\hline Positive & $\begin{array}{l}\text { 7. Joviality } \\
\text { 8. Self-assurance } \\
\text { 9. Attentiveness }\end{array}$ & $\begin{array}{l}\text { happy, joyful, delighted, cheerful, excited, lively, energetic, energised* } \\
\text { proud, strong, confident, bold, daring, fearless, confident* } \\
\text { alert, attentive, concentrating, determined, careful* , motivated* }\end{array}$ \\
\hline Other & $\begin{array}{l}\text { 10. Shyness } \\
\text { 11. Fatigue } \\
\text { 12. Serenity } \\
\text { 13. Surprise }\end{array}$ & $\begin{array}{l}\text { shy, bashful, sheepish, timid } \\
\text { sleepy, tired, sluggish, drowsy } \\
\text { calm, relaxed, at ease, peaceful* } \\
\text { amazed, surprised, astonished }\end{array}$ \\
\hline
\end{tabular}

Table 3: PANAS outcome measure reliabilities

\begin{tabular}{llcl}
\hline Category & Emotion & $\boldsymbol{\alpha}$ & $\mathbf{M N}$ \\
\hline General & 1. Negative & .92 & 2.13 \\
& 2. Positive & .91 & 3.10 \\
\hline Negative & 3. Fear & .92 & 2.22 \\
& 4. Hostility & .90 & 2.11 \\
& 5. Guilt & .91 & 1.71 \\
& 6. Sadness & .89 & 2.48 \\
\hline Positive & 7. Joviality & .95 & 2.99 \\
& 8. Self-assurance & .93 & 2.59 \\
& 9. Attentiveness & .84 & 3.46 \\
\hline Other & 10. Shyness & .84 & 1.90 \\
& 11. Fatigue & .85 & 2.94 \\
& 12. Serenity & .87 & 3.84 \\
& 13. Surprise & .84 & 2.78 \\
\hline
\end{tabular}

\section{Results}

SPSS version 26.0 was utilised to analyse the experimental data. Given the RQ, statistical analyses were performed to examine which media tool would contribute to higher levels of emotion. For each measure of emotion, a one-way ANOVA was performed to examine differences in the emotional arousal levels experienced by the participants assigned to the text, video, and video game conditions.

Out of the 13 emotion outcomes, 6 indicated no differences among the conditions (general negative emotions, fear, guilt, sadness, shyness, and serenity) but 7 emotions did indicate differences between groups (general positive emotions, hostility, joviality, self-assurance, attentiveness, fatigue, and surprise). For a summary of all outcomes, see Tables 4-7. In addition, the text description following the tables further describes the differences among groups that emerged for the 7 emotion outcomes that showed statistically significant results. These results are described to allow readers to see these differences in greater depth.

Table 4: ANOVA comparisons for general emotions; asterisks distinguish $\mathrm{p}$ values: ${ }^{*} \mathrm{p}<.05, * * \mathrm{p}<.01, * * * \mathrm{p}<$ .001

\begin{tabular}{llcccccc}
\hline & & & & & \multicolumn{3}{c}{ Tukey HSD comparisons } \\
\cline { 6 - 9 } Emotion & & $n$ & MN & SD & Text & Video & Game \\
\hline 1. Negative & Text & 47 & 2.14 & 1.03 &. & & \\
& Video & 48 & 1.85 & 1.08 & .439 &. & \\
& Game & 58 & 2.36 & 1.27 & .589 & .062 &. \\
\hline 2. Positive & Text & 47 & 3.17 & 1.16 &. & & \\
& Video & 48 & 2.51 & 1.25 & $.02^{*}$ &. & \\
& Game & 58 & 3.53 & 1.16 & .270 & $.000^{* * *}$ &. \\
\hline
\end{tabular}


Table 5: ANOVA comparisons for negative emotions; asterisks distinguish $\mathrm{p}$ values: ${ }^{*} \mathrm{p}<.05,{ }^{*} \mathrm{p}<.01, * * * \mathrm{p}<$ .001

\begin{tabular}{llcccccc}
\hline & & & & & \multicolumn{3}{c}{ Tukey HSD comparisons } \\
\cline { 6 - 8 } Emotion & & $n$ & $M N$ & SD & Text & Video & Game \\
\hline 3. Fear & Text & 47 & 2.30 & 1.28 &. & & \\
& Video & 48 & 1.87 & 1.19 & .245 &. & \\
& Game & 58 & 2.44 & 1.43 & .853 & .069 &. \\
\hline 4. Hostility & Text & 47 & 1.92 & 0.96 &. & & \\
& Video & 48 & 1.91 & 0.99 & .998 &. & \\
& Game & 58 & 2.42 & 1.33 & .062 & $.051^{*}$ &. \\
\hline 5. Guilt & Text & 47 & 1.70 & 0.80 &. & & \\
& Video & 48 & 1.53 & 0.96 & .674 &. & \\
& Game & 58 & 1.87 & 1.10 & .657 & .183 &. \\
\hline 6. Sadness & Text & 47 & 2.77 & 1.54 &. & & \\
& Video & 48 & 2.40 & 1.30 & .390 &. & \\
& Game & 58 & 2.31 & 1.24 & .210 & .943 &. \\
\hline
\end{tabular}

Table 6: ANOVA comparisons for positive emotions; asterisks distinguish $\mathrm{p}$ values: ${ }^{*} \mathrm{p}<.05,{ }^{* *} \mathrm{p}<.01, * * * \mathrm{p}<$ .001

\begin{tabular}{llcccccc}
\hline & & & & \multicolumn{5}{c}{ Tukey HSD comparisons } \\
\cline { 7 - 9 } Emotion & & $n$ & $M N$ & $S D$ & Text & Video & Game \\
\hline 7. Joviality & Text & 47 & 3.26 & 1.38 &. & & \\
& Video & 48 & 2.42 & 1.37 & $.011^{*}$ &. & \\
& Game & 58 & 3.24 & 1.43 & .998 & $.008^{* *}$ &. \\
\hline 8. Self-assurance & Text & 47 & 2.66 & 1.18 &. & & \\
& Video & 48 & 1.97 & 1.27 & $.030^{*}$ &. & \\
& Game & 58 & 3.04 & 1.47 & .329 & $.000^{* * *}$ &. \\
\hline 9. Attentiveness & Text & 47 & 3.42 & 1.11 &. & & \\
& Video & 48 & 2.94 & 1.22 & .135 &. & \\
& Game & 58 & 3.91 & 1.30 & .105 & $.000^{* * *}$ &. \\
\hline
\end{tabular}

Table 7: ANOVA comparisons for affective states; asterisks distinguish $p$ values: ${ }^{*} p<.05, * * p<.01, * * * p<.001$

\begin{tabular}{llllrrrr}
\hline & & & & \multicolumn{4}{c}{ Tukey HSD comparisons } \\
\cline { 6 - 9 } Emotion & & $n$ & $M N$ & SD & Text & Video & Game \\
\hline 10. Shyness & Text & 47 & 1.91 & .98 &. & & \\
& Video & 48 & 1.72 & 1.21 & .670 &. & \\
& Game & 58 & 2.03 & 1.14 & .849 & .320 &. \\
\hline 11. Fatigue & Text & 47 & 2.82 & 1.21 &. & & \\
& Video & 48 & 3.39 & 1.52 & .110 &. & \\
& Game & 58 & 2.65 & 1.38 & .805 & $.018^{*}$ &. \\
\hline 12. Serenity & Text & 47 & 4.09 & 1.42 &. & & \\
& Video & 48 & 3.86 & 1.48 & .720 &. & \\
& Game & 58 & 3.63 & 1.46 & .249 & .706 &. \\
\hline 13. Surprise & Text & 47 & 2.87 & 1.38 &. & & \\
& Video & 48 & 2.17 & 1.33 & $.038^{*}$ &. & \\
& Game & 58 & 3.20 & 1.45 & .452 & $.001 * *$ &. \\
\hline
\end{tabular}

For general positive emotions, emotional arousal was lowest in the video condition $(M=2.51, S D=1.25)$, somewhat higher in the text condition $(M=3.17, S D=1.16)$, and highest in the video game condition $(M=$ 3.53, $S D=1.16)$. Differences among the conditions were significant overall, $F(2,150)=9.861, p<.001$. However, a post hoc Tukey analysis test indicated that despite significant differences between the text and the video $(p=.02)$ and the video and the video game $(p<.001)$, there was no significant difference between the text and the video game $(p=.27)$. Positive emotions appeared relatively equal in the text and the video game conditions but lower in the video condition.

For hostility, emotional arousal was lowest in the video condition $(M=1.91, S D=.99)$, somewhat higher in the text condition $(M=1.92, S D=.96)$, and highest in the video game condition $(M=2.42, S D=1.33)$. Differences among the conditions were significant overall, $F(2,150)=3.706, p=.027$. However, a post hoc Tukey analysis test indicated that the difference between the video game and the video only approached significance $(p=$ 
.051) and that there was no significant difference between the text and the video $(p=.998)$ or the text and the video game $(p=.062)$. Therefore, hostility in the video game condition was only slightly elevated in comparison to the video condition.

For joviality, emotional arousal was lowest in the video condition $(M=2.42, S D=1.37)$, somewhat higher in the game condition $(M=3.24, S D=1.43)$, and highest in the text condition $(M=3.26, S D=1.38)$. Differences among the conditions were significant overall, $F(2,150)=5.814, p=.004$. A post hoc Tukey analysis test indicated that despite significant differences between the text and the video $(p=.011)$, and the video and the video game $(p=.008)$, there was no significant difference between the text and the video game $(p=.998)$. Joviality appeared relatively equally higher in the text and the video game conditions and again lower in the video condition.

For self-assurance, emotional arousal was lowest in the video condition $(M=1.97, S D=1.27)$, somewhat higher in the text condition $(M=2.66, S D=1.18)$, and highest in the video game condition $(M=3.04, S D=$ 1.47). Differences among the conditions were significant overall, $F(2,150)=8.689, p<.001$. A post hoc Tukey analysis test indicated that despite significant differences between the text and the video $(p=.03)$ and the video and the video game $(p<.001)$, there was no significant difference between the text and the video game $(p=.329)$. Self-assurance appeared relatively equally higher in the text and the video game conditions and again lower in the video condition.

For attentiveness, emotional arousal was lowest in the video condition $(M=2.94, S D=1.22)$, somewhat higher in the text condition $(M=3.42, S D=1.11)$, and highest in the video game condition $(M=3.91, S D=1.30)$. Differences among the conditions were significant overall, $F(2,150)=8.357, p<.001$. A post hoc Tukey analysis test indicated that despite significant differences between the video and the video game $(p<.001)$, there was no significant difference between the text and the video $(p=.135)$ and the text and the video game $(p=.105)$. Therefore, attentiveness was higher overall for the video game condition, although this result seemed to overlap with the text condition.

For fatigue, emotional arousal was lowest in the video game condition $(M=2.65, S D=1.38)$, somewhat higher in the text condition $(M=2.82, S D=1.21)$, and highest in the video condition $(M=3.39, S D=1.52)$. Differences among the conditions were significant overall, $F(2,150)=4.050, p=.019$. A post hoc Tukey analysis test indicated that despite significant differences between the video and the video game $(p=.018)$, there was no significant difference between the text and the video $(p=.110)$ and the text and the video game $(p=.805)$. Therefore, fatigue was lower overall for the video game condition, although this result again seemed to overlap with the text condition.

For surprise, emotional arousal was lowest in the video condition $(M=2.17, S D=1.33)$, somewhat higher in the text condition $(M=2.87, S D=1.38)$, and highest in the video game condition $(M=3.20, S D=1.45)$. Differences among the conditions were significant overall, $F(2,150)=7.436, p=.001$. A post hoc Tukey analysis test indicated that despite significant differences between the text and the video $(p=.038)$ and the video and the video game $(p<.001)$, there was no significant difference between the text and the video game $(p=.452)$. Surprise appeared relatively equally higher in the text and the video game conditions and again lower in the video condition.

\section{Discussion}

This experiment examined three types of mass media tools: text, video, and video game to better understand the mechanisms (comparing media attributes of textuality, channel, interactivity, and control) that might contribute to positive emotional outcomes related to teaching and learning. Of the 13 emotion outcomes examined, 6 indicated no differences between the conditions (general negative emotions, fear, guilt, sadness, shyness, and serenity), but 7 emotions did indicate differences in groups (general positive emotions, hostility, joviality, self-assurance, attentiveness, fatigue, and surprise). Although, it is worth mentioning that on a sevenpoint scale, the participants indicated relatively low emotional intensity levels overall, all averaging less than four. This finding might be explained by the fact that the story's overall content did not contribute to intense emotional responses. 
Still, a consistent pattern emerged for differences among the conditions. When examining the 5 positive emotional aspects of general positive emotions, joviality, self-assurance, attentiveness, and surprise, it is clear that both the text and the video game conditions were better at contributing to higher levels of positive emotional arousal than just the video alone. When examining this outcome through a media-attributes lens, previously this study established that the video condition featured attributes low in textuality, high in sensory channel, low in interactivity, and low in control. Therefore, these attributes together overall lead to a less positive experience. These findings align to some degree with past research results as Plass et al. (2019) also found that simple 2D characters on a screen alone did not influence the positive emotion of happiness.

In addition, results also indicated that participants experienced somewhat higher levels of hostility in the video game condition in comparison to the video condition (although these results overlapped with the text condition and were only approaching significance). Still, speculation suggests that this result is likely due to the higher degree of interactivity in the video game. This attribute likely enabled the participants to feel more intense arousal due to the actions afforded by the video game condition (Klimmt et al., 2010), allowing the participants to experience the story from the character's perspective and likely increasing their perceptions of hostility to some degree.

Lastly, feelings of fatigue were generally lower in the video game condition compared with the video condition (although the text condition had some overlap between the two again). This finding makes sense as past research indicates that video games can create motivational experiences (Ryan, Rigby and Przybylski, 2006), and given the attributes identified in this study, this motivational quality is likely afforded by a video game's higher degrees of sensory channel, interactivity, and control that help reduce feelings of fatigue. Overall, this study's implications suggest that when content is controlled but delivered via different mediated learning technology tools, video games and texts are more likely to trigger higher levels of emotional arousal than videos.

\subsection{Limitations and future research}

This study was subject to three primary limitations. First, the study was quantitative, so the authors could not understand the detailed reasons for the differences between the positive emotions in the video game and the text conditions or the lower levels of such emotions in the video condition. It is necessary to further explore why this was the case. For example, a thematic analysis using open-ended emotion-related questions may provide deeper insights into how students respond to mediated e-learning experiences. In addition, future research may also consider exploring user preferences, engagement, and other mediated factors, including pre-existing video game usage. Using research methods such as interviews and the "think aloud" protocol (to examine player experiences) may also be used to derive the tacit assumptions that could teach researchers more about emotional arousal and differences across media tools.

Second, the measures of emotion were self-reported. Future research could adopt more objective measures to examine emotional arousal to triangulate the findings. For instance, objective assessment methods using noninvasive physiological responses, such as heart rate variability and galvanic skin conductance using a wearable device, could track the participants' emotional arousal during tasks.

Lastly, the media experiences in this study triggered relatively low levels of emotional intensity. To better examine this phenomenon, using media content that would more likely result in higher emotional arousal could better teach researchers about differences among mediated learning tools. Although this research provided an initial step as a comparison study examining levels of emotion through the lens of media attributes, much work remains for researchers to explore in this area.

\subsection{Final thoughts}

This study sought to answer the research question, which media tool would contribute to higher levels of positive emotion in an e-learning context? Key findings showed that both a text and a video game contributed to similar higher levels of positive emotional arousal in comparison to watching a video alone. When examining these findings through a mixed attributes lens, we can identify the more precise mechanisms that may be driving these outcomes.

In the case of the text and the video game used in this study, both were more likely to facilitate a higher level of control over the media experience in comparison to the video condition. The text enabled more control 
because students could adjust the pausing and pacing of their reading based on their individual needs. Similarly, the video game enabled more control because students could make decisions in the game that ultimately impacted their playthrough experience. Therefore, providing users with more control and autonomy during e-learning may be more likely to better facilitate student emotion.

In our modern teaching landscape today, instructors have numerous options available in designing a course and in selecting what kind of media will best deliver meaningful learning experiences to students. While more work is needed to further examine the effects of teaching medium on outcomes related to student learning, these results may be useful to educators considering both the affordances and limitations of different kinds of media tools and the role they may play in the academic environment.

\section{References}

Adams, A., 2016. The effect of varying media attributes on emotion and introspection in mediated learning contexts. In: NCA (National Communication Association), 102 ${ }^{\text {nd }}$ Conference. Philadelphia, PA, 10-13 November 2016.

Althoff, T., White, R.W. and Horvitz, E., 2016. Influence of Pokémon Go on physical activity: Study and implications. Journal of Medical Internet Research, [e-journal] 18(12), p.e315. http://dx.doi.org/10.2196/jmir.6759

Benschoter, R.P. and Charles, D.C., 1957. Retention of classroom and television learning. Journal of Applied Psychology, 41(4), pp.253-256. https://doi.org/10.1037/h0043126

Bigge, M.L., 1982. Learning theories for teachers. New York: Harper and Row.

Bittner, W.S. and Mallory, M.F., 1933. University teaching by mail: A survey of correspondence instruction conducted by American universities. New York: Macmillan.

Brown, J.S., 2006. New learning environments for the 21st century: Exploring the edge. Change: The Magazine of Higher Learning, 38(5), pp.18-24. https://doi.org/10.3200/CHNG.38.5.18-24

Connolly, T.M., Boyle, E.A., MacArthur, E., Hainey, T. and Boyle, J.M., 2012. A systematic literature review of empirical evidence on computer games and serious games. Computers and Education, 59(2), pp.661-686. https://doi.org/10.1016/i.compedu.2012.03.004

Coulter, R., Klopfer, E., Perry, J. and Sheldon, J., 2012. Discovering familiar places: Learning through mobile place-based games. In: C. Steinkuehler, K. Squire and S. Barab, eds. 2012., Games, learning, and society: Learning and meaning in the digital age. New York, NY: Cambridge University Press. pp.327-354.

Detenber, B.H. and Reeves, B., 1996. A bio-informational theory of emotion: motion and image size effects on viewers. Journal of Communication, 46(3), pp.66-84. https://doi.org/10.1111/j.1460-2466.1996.tb01489.x

Detenber, B.H. and Lang, A., 2010. The influence of form and presentation attributes of media on emotion. In: K. Doveling, C. von Scheve and E. Konijin, eds. 2010. The Routledge Handbook of Emotions and Mass Media. New York: Routledge. pp.275-293.

Dunbar, N.E., Wilson, S., Adame, B., Elizondo, J., Jensen, M.L., Miller, C., Allums. A., Seltsam, T., Bessarabova, E., Vincent, C., Straub, S., Ralston, R., Dulawan, C.L., Ramirez, D., Squire, K., Valacich, J. and Burgoon, J.K., 2013. The development of a training game for the mitigation of cognitive bias: The case study of MACBETH. International Journal of Game-Based Learning, 3(4), pp.7-26. https://doi.org/10.4018/ijgbl.2013100102

Eveland Jr, W.P., 2003. A "mix of attributes" approach to the study of media effects and new communication technologies. Journal of Communication, 53(3), pp.395-410. https://doi.org/10.1111/j.1460-2466.2003.tb02598.x

Eveland Jr, W. P. and Dunwoody, S., 2001. User control and structural isomorphism or disorientation and cognitive load? Learning from the web versus print. Communication Research, 28(1), pp.48-78. https://doi.org/10.1177\%2F009365001028001002

Ferdig, R.E., Baumgartner, E. and Gandolfi, E. eds., 2016. Teaching the game: A collection of syllabi for game design, development, and implementation (Vol. 1). Pennsylvania: ETC Press. Available at https://press.etc.cmu.edu/index.php/product/teaching-the-game-volume-1/ [Accessed 23 August 2021]

Ferguson, C., van den Broek, E.L. and van Oostendorp, H., 2020. On the role of interaction mode and story structure in virtual reality serious games. Computers and Education, [e-journal] 143, pp.1-10. https://doi.org/10.1016/i.compedu.2019.103671

Flowerday, T. and Schraw, G., 2000. Teacher beliefs about instructional choice: A phenomenological study. Journal of Educational Psychology, 92(4), pp.634-645. https://doi.org/10.1037/0022-0663.92.4.634

Frijda, N.H., 1986. The emotions. New York: Cambridge University Press.

Galloway, A.R., 2006. Gaming: Essays on algorithmic culture (Vol. 18). Minnesota: U of Minnesota Press.

Grace, L., 2020. Doing things with games: Social impact through play. Florida: CRC Press.

Heumos, T. and Kickmeier-Rust, M.D., 2020. Using game-based training to reduce media induced anxiety in young children-A pilot study on the basis of a game-based app. (MARTY). Electronic Journal of e-Learning, 18(3), pp.207218. https://doi.org/10.34190/EJEL.20.18.3.001

Hilton, M. and Honey, M.A. eds., 2011. Learning science through computer games and simulations. Washington, D. C.: The National Academies Press.

Howland, J.L. and Moore, J.L., 2002. Student perceptions as distance learners in Internet-based courses. Distance Education, 23(2), pp.183-195. https://doi.org/10.1080/0158791022000009196 
Jackson, L., 2011. Steam for schools - Learning with portals. G4 the Feed. Available at: http://www.learnwithportals.com [Accessed 23 August 2021]

Jacobs, R.S., 2021. Winning over the players: Investigating the motivations to play and acceptance of serious games. Media and Communication, 9(1), pp.28-38. https://doi.org/10.17645/mac.v9i1.3308

Juul, J., 2005. Video games and the classic model. Half Real--video games between real rules and fictional worlds. Cambridge, MA: The MIT Press.

Kaplan, A.M. and Haenlein, M., 2010. Users of the world, unite! The challenges and opportunities of social media. Business Horizons, [e-journal] 53(1), pp.59-68. https://doi.org/10.1016/j.bushor.2009.09.003

Klimmt, C., Hefner, D., Vorderer, P., Roth, C. and Blake, C., 2010. Identification with video game characters as automatic shift of self-perceptions. Media Psychology, 13(4), pp.323-338. https://doi.org/10.1080/15213269.2010.524911

Kohn, A., 1993. Choices for children: Why and how to let students decide. Phi Delta Kappan, 75(1), pp.18-21. Available at: https://www.myececlass-basics.com/uploads/1/1/9/4/119403380/choices for children.pdf [Accessed 23 August 2021]

Koster, R., 2004. A theory of fun for game design. Scottsdale, AZ: Paraglyph Press.

Lang, A., 2006. Using the limited capacity model of motivated mediated message processing to design effective cancer communication messages. Journal of Communication, 56(s1), pp.57-80. https://doi.org/10.1111/j.14602466.2006.00283.x

Lang, P.J., Greenwald, M.K., Bradley, M.M. and Hamm, A., 1993. Looking at pictures: Affective, facial, visceral, and behavioral reactions. Psychophysiology, 30(3), pp.261-273. https://doi.org/10.1111/i.1469-8986.1993.tb03352.x

Lazarus, R.S., 1991. Emotion and adaptation. New York: Oxford University Press.

Mandaro, L., 2014. How to get more girls to code: Use Frozen's Elsa. USA Today. Available at: https://www.usatoday.com/story/tech/2014/11/19/disney-codeorg-frozen-girls-computer-programming/19260097/ [Accessed 23 August 2021]

Mayer, R.E., 2014. Computer games for learning: An evidence-based approach. Cambridge: MIT Press.

McGonigal, J., 2011. Reality is broken. New York: Penguin.

McLuhan, M., 1964. Understanding media: The extensions of man. New York: McGraw Hill.

Miller, C.H., Dunbar, N.E., Jensen, M.L., Massey, Z., Nicholls, S.B., Lee, Y.H., Anderson, C., Adams, A.S., Cecena, J.E., Thompson, W.M. and Wilson, S.N., 2019. Training law enforcement officers to identify reliable deception cues with a serious digital game. International Journal of Game-Based Learning, 9(3), pp.1-22. https://doi.org/10.4018/IJGBL.2019070101

Moore, M.G. ed., 2013. Handbook of distance education. New York and London: Routledge.

Nabi, R.L. and Green, M.C., 2015. The role of a narrative's emotional flow in promoting persuasive outcomes. Media Psychology, 18(2), pp.137-162. https://doi.org/10.1080/15213269.2014.912585

Newhagen, J.E. and Rafaeli, S., 1996. Why communication researchers should study the Internet: A dialog. Journal of Communication, [e-journal] 46(1), pp.4-13. https://doi.org/10.1111/j.1083-6101.1996.tb00172.x

Pivec, M., 2007. Editorial: Play and learn: Potentials of game-based learning. British Journal of Educational Technology, 38(3), pp.387-393. https://doi.org/10.1111/j.1467-8535.2007.00722.x

Plass, J.L., Homer, B.D., MacNamara, A., Ober, T., Rose, M.C., Pawar, S., Hovey, C.M. and Olsen, A., 2019. Emotional design for digital games for learning: The effect of expression, color, shape, and dimensionality on the affective quality of game characters. Learning and Instruction, [e-journal] 70, pp.1-13. https://doi.org/10.1016/i.learninstruc.2019.01.005

Prensky, M., 2005. Computer games and learning: Digital game-based learning. In: J. Raessens and J. Goldstein, eds. 2005 (Vol. 18). Handbook of Computer Games Studies. Cambridge, MA: The MIT Press. pp. 97-122.

Reeves, B., Lang, A., Kim, E. and Tartar, D., 1999. The effects of screen size and message content on attention and arousal. Media Psychology, 1(1), pp.49-67. https://doi.org/10.1207/s1532785xmep0101 4

Ryan, R.M., Rigby, C.S. and Przybylski, A., 2006. The motivational pull of video games: A self-determination theory approach. Motivation and Emotion, 30(4), pp.344-360. https://doi.org/10.1007/s11031-006-9051-8

Sailer, M. and Homner, L., 2020. The gamification of learning: A meta-analysis. Educational Psychology Review, [e-journal] 32, pp.77-112. https://doi.org/10.1007/s10648-019-09498-w

Schrier, K.S. ed., 2016. Learning, education, and games (Vol. 2). Pennsylvania: ETC Press. Available at https://press.etc.cmu.edu/index.php/product/learning-education-and-games-volume-two-bringing-games-intoeducational-contexts/ [Accessed 23 August 2021]

Schrier, K.S. ed., 2019. Learning, education, and games (Vol. 3). Pennsylvania: ETC Press. Available at https://press.etc.cmu.edu/index.php/product/learning-education-games-volume-3/ [Accessed 23 August 2021]

Shaffer, D.W., 2012. Models of situated action: Computer games and the problem of transfer. In: C. Steinkuehler, K. Squire and S. Barab, eds. 2012. Games, learning, and society: Learning and meaning in the digital age. New York, NY: Cambridge University Press. pp.403-431.

Squire, K., 2003. Video games in education. International Journal of Intelligent Simulations and Gaming, 2(1), pp.49-62. Available at: http://citeseerx.ist.psu.edu/viewdoc/download?doi=10.1.1.543.5729\&rep=rep1\&type=pdf [Accessed 23 August 2021]

Squire, K., 2011. Video games and learning. New York: Teachers College Press. 
Tamborini, R. and Skalski, P., 2006. The role of presence in the experience of electronic games. In: P. Vorderer and J. Bryant, eds. 2006. Playing video games: Motives, responses, and consequences. New Jersey: Lawrence Erlbaum Associates Publishers. pp.225-240.

Upchurch, R.W. and Wildermuth, S., 2014. Gamification in education: Past and present patterns, and future trends. In: NCA (National Communication Association), 100 th Conference. Chicago, IL, 20-23 November 2014.

Watson, D. and Clark, L.A., 1999. The PANAS-X: Manual for the positive and negative affect schedule-expanded form. lowa: The University of lowa. https://doi.org/10.17077/48vt-m4t2

Yee, N., 2009. Befriending ogres and wood-elves: Relationship formation and the social architecture of Norrath. Game Studies, [e-journal] 9(1). Available at: http://gamestudies.org/0901/articles/yee [Accessed 23 August 2021]

Yee, K., 2013. Pedagogical gamification: Principles of video games that can enhance teaching. To Improve the Academy: Resources for Faculty, Instructional, and Organizational Development, 32(1), pp.335-349. https://doi.org/10.1002/j.2334-4822.2013.tb00714.x 